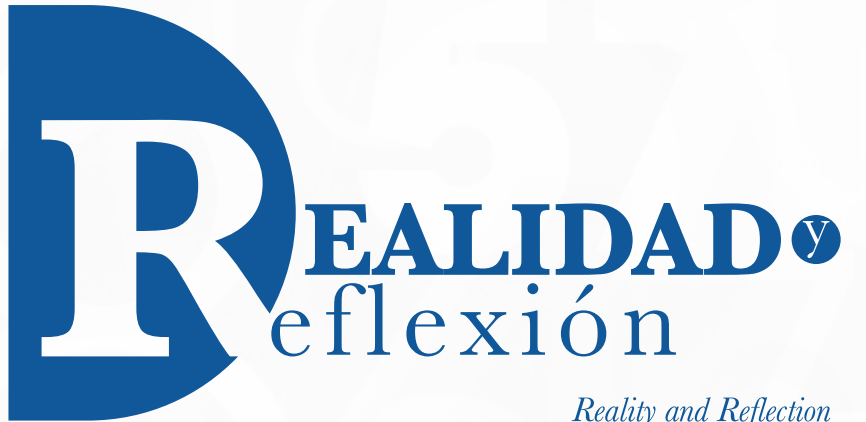

ISSN 1992-6510

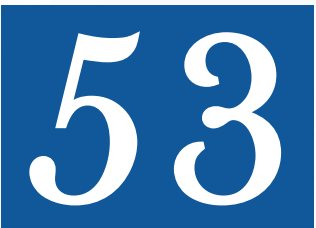

Año 21, N 53, San Salvador, El Salvador, Centroamérica. Revista Semestral Enero-Junio 2021

YEAR 21, N53, SAN SALVADOR, EL SALVADOR, CENTRAL AMERICA. SEMESTRAL JOURNAL JANUARY-JUNE 2021

\title{
Apoyando el desarrollo de la competencia mirar profesionalmente del futuro profesorado de matemáticas: Práctica e investigación
}

Supporting the development of future mathematics teachers' professional noticing competence: Practice and research

Ceneida Fernández Departamento de Innovación y Formación Didáctica. Universidad de Alicante (España) ceneida.fernandez@ua.es

Recibido: 3 de julio de 2020 Aceptado: 1 de octubre de 2020 DOI: $10.5377 /$ ryr.v53i53.10887

(ब⿻肀 


\section{RESUMEN}

La competencia docente mirar profesionalmente es vista como una componente de la práctica profesional del profesor de matemáticas, $y$, por tanto, ha sido identificada como una competencia docente importante a desarrollar en los programas de formación de profesorado. El grupo GIDIMAT de la Universidad de Alicante (España) se ha centrado en investigar el desarrollo de esta competencia en futuro profesorado de educación infantil, primaria y secundaria. Para el desarrollo de esta competencia se diseñan entornos de aprendizaje específicos dentro de los programas de formación. En este artículo se presenta un ejemplo de entorno de aprendizaje y los principales resultados obtenidos del desarrollo de la competencia. El artículo se divide en cinco secciones. En la primera, se revisan estudios centrados en la competencia mirar profesionalmente el pensamiento matemático de los estudiantes y su relación con los dominios de conocimiento. En la segunda, se presenta una revisión de estudios centrados en su desarrollo. En la tercera sección, se presenta la metodología usada por el grupo GIDIMAT a través de ciclos de investigación en el diseño de entornos de aprendizaje. Además, se presentan las características de los entornos y viñetas usadas. En la cuarta sección se muestran resultados obtenidos relacionados con la caracterización de grados de desarrollo de la competencia docente. Finalmente, se discuten algunas implicaciones y trabajos futuros.

Palabras Clave: Mirar profesionalmente, formación de profesorado de matemáticas, competencia, representaciones de la práctica, tarea profesional.

\section{ABSTRACT}

Professional noticing competence is seen as a component of the mathematics teacher professional practice and, therefore, it has been identified as an important teaching competence to be developed in teacher training programs. The GIDIMAT group of the University of Alicante (Spain) has focused on investigating the development of this competence in pre-service pre-school and primary school teachers and prospective secondary school mathematics teachers. For the development of this competence, specific learning environments are designed within the teacher training programs. In this paper, an example of a learning environment and the main results obtained related to the competence development are presented. The article is divided into five sections. In the first, studies focused on the competence of noticing students' mathematical thinking and its relationship with the domains of knowledge have been reviewed. In the second, a review of studies focused on the competence development is shown. In the third section, the methodology used by the GIDIMAT group through research cycles in the design of learning environments is presented. Moreover, the characteristics of these learning environments and vignettes used are discussed. The fourth section contains the results obtained related to the characterization of degrees of the competence development. Finally, some implications and further research are discussed.

Keywords: Professional noticing, primary and secondary school mathematics teacher training, competence, representation of practice, professional task. 
El conocimiento profesional del profesorado necesario para enseñar matemáticas se considera que está integrado por diferentes dominios. Desde diferentes perspectivas teóricas se han identificado diferentes dominios de conocimiento necesarios para enseñar matemáticas (Carrillo, Climent, Contreras y Muñoz-Catalán, 2013; Godino, Batenero y Font, 2007; Hill, Ball y Schilling, 2008; Rowland, Hutckstep y Thwaites, 2005; Shulman, 1986). Sin embargo, el rasgo que caracteriza el conocimiento profesional del profesorado no está solo en lo que conoce (dominios) sino en su uso (Eraut, 1996). Desde esta perspectiva se subraya la importancia del uso del conocimiento en la resolución de las situaciones problemáticas generadas en su actividad profesional de enseñar matemáticas. Llinares (2013) identifica tres sistemas de actividades que articulan la práctica del profesorado de matemáticas tanto de educación infantil, primaria y secundaria: (i) analizar, diagnosticar y dotar de significado a las producciones matemáticas del alumnado; (ii) seleccionar y diseñar tareas matemáticas adecuadas y (iii) dotar de sentido y gestionar la comunicación matemática en el aula.

En este contexto, la competencia docente mirar profesionalmente (Mason, 2002) es vista como una componente de la práctica profesional del profesorado de matemáticas, $\mathrm{y}$, por tanto, ha sido identificada como una competencia docente importante a desarrollar en el futuro profesorado de educación infantil, primaria y secundaria (Fernández y Choy, 2020; Fernández, Sánchez-Matamoros, Valls y Callejo, 2018; Llinares, Ivars, Buforn, y Groenwald, 2019; Schack, Fisher y Wilhelm, 2017). Mirar profesionalmente las situaciones de enseñanza-aprendizaje de las matemáticas permite al futuro profesorado identificar aspectos relevantes en una situación, usar el conocimiento para interpretarlos y justificar las decisiones de enseñanza (van Es y Sherin, 2002). Por tanto, una mirada profesional distingue a un profesional o experto en una determinada área de alguien que no lo es por su capacidad de ver ciertos fenómenos de una manera particular (Goodwin, 1994). En el caso particular del profesorado de matemáticas, la mirada profesional les permite identificar aspectos relevantes en una situación de enseñanza-aprendizaje que otras personas, no profesionales, serían incapaces de identificar (Roller, 2016) y razonar sobre la situación usando un conocimiento profesional sobre las matemáticas y sobre la enseñanza y aprendizaje de las matemáticas.

Este artículo se divide en cinco secciones. En la primera, se revisan estudios centrados en un aspecto particular de la competencia, en la competencia mirar profesionalmente el pensamiento matemático de los estudiantes y su relación con los dominios de conocimiento. En la segunda, se presenta una revisión de estudios centrados en el desarrollo de esta competencia en los programas de formación de profesorado de matemáticas. En la tercera sección, se presenta la metodología usada por el grupo GIDIMAT de la Universidad de Alicante a través de ciclos de investigación en el diseño de entornos de aprendizaje. En esta sección se presentan las características de los entornos de aprendizaje y viñetas usadas, así como ejemplos de estos. En la cuarta sección se muestran resultados obtenidos relacionados con la caracterización de grados de desarrollo de la competencia docente en el futuro profesorado de infantil, primaria y secundaria. En la quinta sección se discuten algunas implicaciones y trabajos futuros. 


\section{La Competencia Mirar Profesionalmente el Pensamiento Matemático de los Estudiantes}

Desde la NCTM (2014) se ha propuesto un cambio en la manera de afrontar las interacciones con los estudiantes en las aulas subrayando la necesidad de que el profesorado sea flexible para atender las necesidades cognitivas de los estudiantes. Este cambio requiere que sean capaces de analizar e interpretar lo que ocurre en sus clases y así tomar decisiones de enseñanza efectivas. Este contexto, centra la atención en un aspecto particular de la competencia docente, en mirar profesionalmente el pensamiento matemático de los estudiantes. Jacobs, Lamb y Philipp (2010) definen mirar profesionalmente el pensamiento matemático de los estudiantes como tres destrezas que están interrelacionadas: (i) atender las estrategias de los estudiantes identificando detalles matemáticos importantes, (ii) interpretar la comprensión de los estudiantes con base en los detalles matemáticos identificados y (iii) decidir cómo responder atendiendo la comprensión de los estudiantes. Esta competencia implica que el profesorado use su conocimiento matemático y conocimiento sobre la enseñanza y aprendizaje de las matemáticas para atender, interpretar y decidir (Brown, Fernández, Helliwell y Llinares, 2020; Thomas, Jong, Fisher y Schack, 2017). La Figura 1 relaciona los dominios de conocimientos necesarios para enseñar matemáticas del modelo MKT (Mathematical Knowledge for Teaching; Hill et al., 2008) y las destrezas de mirar profesionalmente el pensamiento matemático del estudiante.

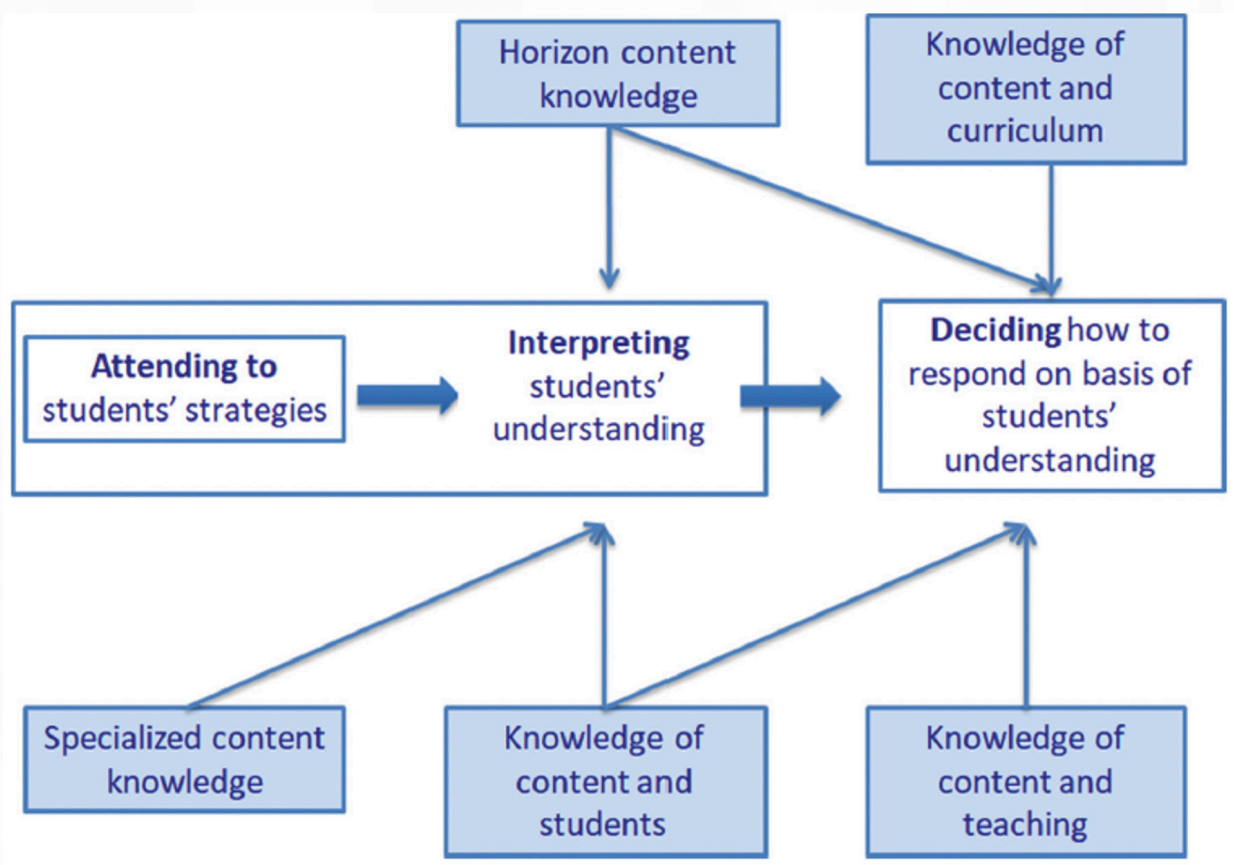

Figura 1. Relación MKT y la competencia mirar profesionalmente el pensamiento matemático del estudiante (Brown et al., 2020, p. 352). 
Esta competencia proporciona al profesorado una estructura para actuar en situaciones de enseñanza y aprendizaje como interpretar la comprensión matemática de los estudiantes y decidir cómo continuar la instrucción para que los estudiantes progresen en su comprensión. Por tanto, esta competencia relaciona la teoría (conocimiento) con la práctica de aula.

Una mirada profesional permitiría al futuro profesorado identificar los elementos matemáticos importantes en la actividad de comparar dos fracciones propias mostrada en la figura 2 y en las respuestas de los estudiantes a esta actividad. De esta manera, atender a las estrategias de los estudiantes implica identificar elementos matemáticos importantes en sus respuestas. Para identificar los elementos matemáticos, el futuro profesorado necesita usar su conocimiento sobre las fracciones. Así, el estudiante 1 representa gráficamente las fracciones, pero no usa "todos" iguales para comparar las fracciones. E1 estudiante 2, representa gráficamente las fracciones y las compara correctamente usando "todos" iguales. El estudiante 3, identifica la relación inversa entre el número de partes y el tamaño de las partes, y usa su conocimiento sobre esta relación para comparar ambas fracciones "a mayor número de divisiones del todo, cada parte es más pequeña”. Para interpretar la comprensión de cada uno de los estudiantes, el futuro profesorado debe usar y relacionar los detalles matemáticos identificados con su conocimiento sobre cómo el pensamiento matemático del estudiante progresa en la comparación de fracciones. De esta manera, el futuro profesorado podría identificar que cada estudiante muestra características de la comprensión de la comparación de fracciones diferentes. El estudiante 1 tiene dificultades en la comparación al no comprender que para comparar fracciones debe usar el mismo todo. Los estudiantes 2 y 3 comparan las fracciones correctamente, sin embargo, el estudiante 2 lo hace gráficamente usando los mismos todos para comparar y el estudiante 3 muestra evidencias de que comprende la relación inversa cuando compara fracciones. Se necesita por tanto un uso del conocimiento sobre diferentes estrategias, y sobre el origen de los errores más comunes (Specialized Content Knowledge y Knowledge of Content and Students), y un uso del conocimiento sobre cómo los conceptos matemáticos se desarrollan en el tiempo entre los diferentes niveles educativos (Horizon Content Knowledge).

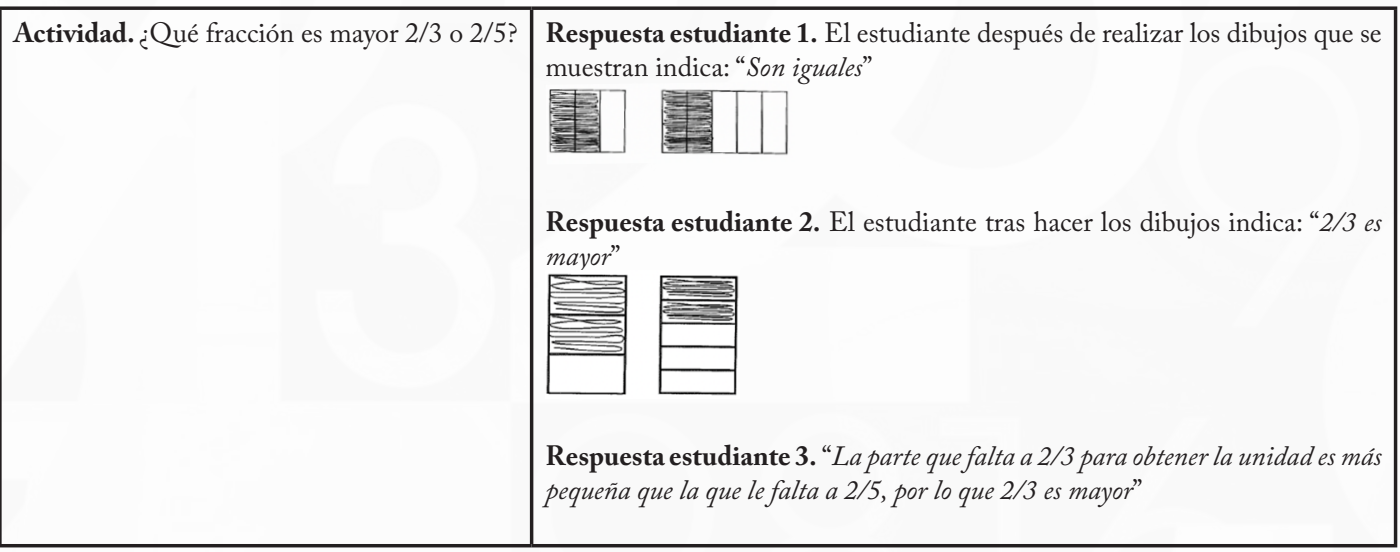

Figura 2. Actividad de comparar fracciones y respuestas de tres estudiantes. 
Por último, para decidir cómo continuar la instrucción con base en la comprensión del estudiante, el futuro profesorado necesita usar su conocimiento sobre qué conceptos son los más fáciles y difíciles de comprender, los errores frecuentes, y las estrategias y representaciones más adecuadas para introducir un concepto (Knowledge of Content and Students and Knowledge of Content and Teaching). Además, necesita usar conocimiento sobre cómo evoluciona el pensamiento matemático del estudiante sobre ese concepto matemático en el tiempo y entre niveles educativos (Horizon Content Knowlege) y conocimiento sobre el diseño de materiales y el uso de recursos (Knowledge of Content and Curriculum). Así, por ejemplo, para el estudiante 1 se podrían proponer actividades con el objetivo de comprender que para comparar fracciones debe usarse la misma unidad bien en contexto continuo (rectángulos o cuadrados, como la respuesta de este estudiante) o en contexto discreto (usando bolitas de papel, por ejemplo).

\section{Desarrollo de la Competencia Mirar Profesionalmente el Pensamiento Matemático de los Estudiantes}

En las últimas décadas se ha desarrollado una agenda de investigación con el objetivo de identificar diferentes contextos favorables para el desarrollo de esta competencia (Fernández y Choy, 2020; Schack et al., 2017; Stahnke, Schueler y Roesken-Winter, 2016), así como indicadores de su desarrollo (Fernández et al., 2018; Llinares, 2019). El uso de registros de la práctica como el análisis de vídeos o el análisis de respuestas escritas de los estudiantes se ha visto como un contexto favorable para su desarrollo (Ivars, Fernández y Llinares, 2020; Sánchez-Matamoros, Fernández y Llinares, 2015; 2019; Schack et al., 2013; van Es y Sherin, 2008). Otros contextos que también favorecen su desarrollo son la escritura de narrativas (Fernández, Llinares y Rojas, 2020; Ivars y Fernández, 2018), los debates online y el feedback del tutor (Fernández, Llinares y Valls, 2012; Ivars y Fernández, 2018; Llinares y Valls, 2010). Estos estudios han mostrado que se puede desarrollar esta competencia en los programas de formación de profesorado de Educación infantil, primaria y secundaria, aunque su desarrollo no es fácil sin unas referencias que guíe al futuro profesorado de matemáticas hacia qué y cómo mirar (Fernández y Choy, 2020; Sztajn y Wilson, 2019).

Una de las herramientas identificadas desde la investigación que puede actuar como guía para el futuro profesorado son las trayectorias hipotéticas de aprendizaje (Clements y Sarama, 2009; Edgington, Wilson, Sztajn y Webb, 2016; Wilson, Sztajn, Edgington y Myers, 2015). Las trayectorias de aprendizaje se han conceptualizado desde diferentes perspectivas (Lobato y Walters, 2017), sin embargo, la característica común es que proporcionan un modelo de progresión del aprendizaje de un concepto matemático particular, es decir, niveles de progresión cada vez más sofisticados en el pensamiento matemático. De acuerdo con Simon (1995), una trayectoria hipotética de aprendizaje (THA) consta de tres componentes: (i) un objetivo de aprendizaje, (ii) un camino hipotético de aprendizaje entendido como una predicción de cómo evoluciona el pensamiento matemático del estudiante (niveles de desarrollo de la comprensión de un determinado concepto) y (iii) un conjunto de actividades que apoyen la comprensión del concepto. Por tanto, las THA pueden proporcionar 
referencias al futuro profesorado para reconocer diferentes niveles en la comprensión de un concepto y de esta manera, proponer actividades que apoyen la progresión en el aprendizaje de los estudiantes, es decir, pueden apoyar el desarrollo de la competencia mirar profesionalmente el pensamiento matemático de los estudiantes.

Estudios previos han mostrado que las THA dotan al futuro profesorado de un lenguaje profesional para describir el pensamiento matemático de los estudiantes y, centran su atención sobre la comprensión matemática de los estudiantes que les ayuda a proporcionar una instrucción apropiada (Sztajn, Confrey, Wilson y Edgington, 2012).

El grupo GIDIMAT del Departamento de Innovación y Formación Didáctica de la Universidad de Alicante (España) se ha centrado en investigar contextos que favorecen el desarrollo de esta competencia en futuro profesorado de educación infantil y primaria y futuro profesorado de matemáticas de educación secundaria dentro de los programas de formación de esta Universidad y en generar indicadores de su desarrollo (revisión en Fernández et al., 2018). En particular, el uso de las THA, el uso de registros de la práctica y el diseño de cuestiones guías están permitiendo caracterizar el desarrollo de la competencia mirar profesionalmente el pensamiento matemático de los estudiantes.

\section{Ciclos de Investigación en el Diseño de Entornos de Aprendizaje}

Las investigaciones del grupo GIDIMAT usan la metodología Design-Based Research (diseño de experimentos de enseñanza) a través de la iteración de ciclos de diseño, implementación en contextos reales, análisis y el rediseño. Los experimentos de enseñanza constituyen el contexto en el que la práctica de formar al profesorado y la investigación sobre el aprendizaje del profesorado se interrelacionan (Llinares, 2014).

Un experimento de enseñanza contempla ciclos de investigación en tres fases (Design-Based Researcher Collective, 2003). La fase 1 es de diseño y planificación de la instrucción donde se fijan los objetivos de aprendizaje que delimitan las metas a alcanzar en el programa de formación del profesorado y se diseñan las tareas para el logro de los objetivos. La fase 2 se corresponde con la implementación de las tareas diseñadas. En la fase 3 se lleva a cabo un análisis retrospectivo en el que los profesores e investigadores observan y analizan la experiencia, apoyando los análisis del aprendizaje del profesorado desde las referencias teóricas sobre cómo entendemos el aprendizaje/desarrollo del profesorado. Este análisis pueda dar lugar a realizar modificaciones en el entorno de aprendizaje inicialmente diseñado (rediseño).

Las investigaciones han aportado características para el diseño de entornos de aprendizaje en estos programas que ayuden a generar oportunidades "desde y para la práctica" al futuro profesorado de matemáticas (Buforn, Llinares, Fernández, Coles y Brown, 2020; Fernández et al., 2012; Fernández, Sánchez-Matamoros, Moreno y Callejo, 2018; Ivars, Buforn y Llinares, 2017; Ivars, Fernández, Llinares y Choy, 2018b; Sánchez- 
Matamoros et al., 2019). Se entiende por entorno de aprendizaje "la conjunción de las tareas diseñadas y la concepción de una determinada manera de usarlas, incluyendo el papel del formador de profesores y los documentos adicionales"(Llinares, 2004, p. 97). Por tarea profesional (o viñeta) nos referiremos al conjunto de registros de la práctica, las preguntas guías que están relacionadas con las actividades que integran la práctica profesional de enseñar matemáticas y la información teórica para interpretar dicho registro y dar respuesta a las preguntas guía. A continuación, se muestran las características de los entornos de aprendizaje y un ejemplo específico de un entorno relacionado con el significado de fracción como parte-todo que forma parte de un programa de formación de profesorado de educación primaria.

\section{Características de los Entornos de Aprendizaje}

Los entornos de aprendizaje son diseñados a partir de diferentes tareas profesionales (viñetas) que giran alrededor del uso de un documento teórico con información que proviene de las investigaciones en Didáctica de la Matemática en forma de trayectoria hipotética de aprendizaje.

En particular, la Figura 3 muestra la distribución de sesiones de un entorno de aprendizaje centrado en el significado de fracción como parte-todo (Ivars, 2018). Este entorno de aprendizaje consta de seis sesiones de 120 minutos de duración cada una. En las tres primeras sesiones se introducen los elementos matemáticos involucrados en la THA mediante la resolución de actividades de fracciones de educación primaria y el visionado y análisis de video-clips en los que se muestran estudiantes de educación primaria resolviendo estas actividades (registro de la práctica). En las tres sesiones siguientes, el futuro profesorado resuelve tres tareas profesionales (viñetas) usando la información teórica proporcionada en la THA.

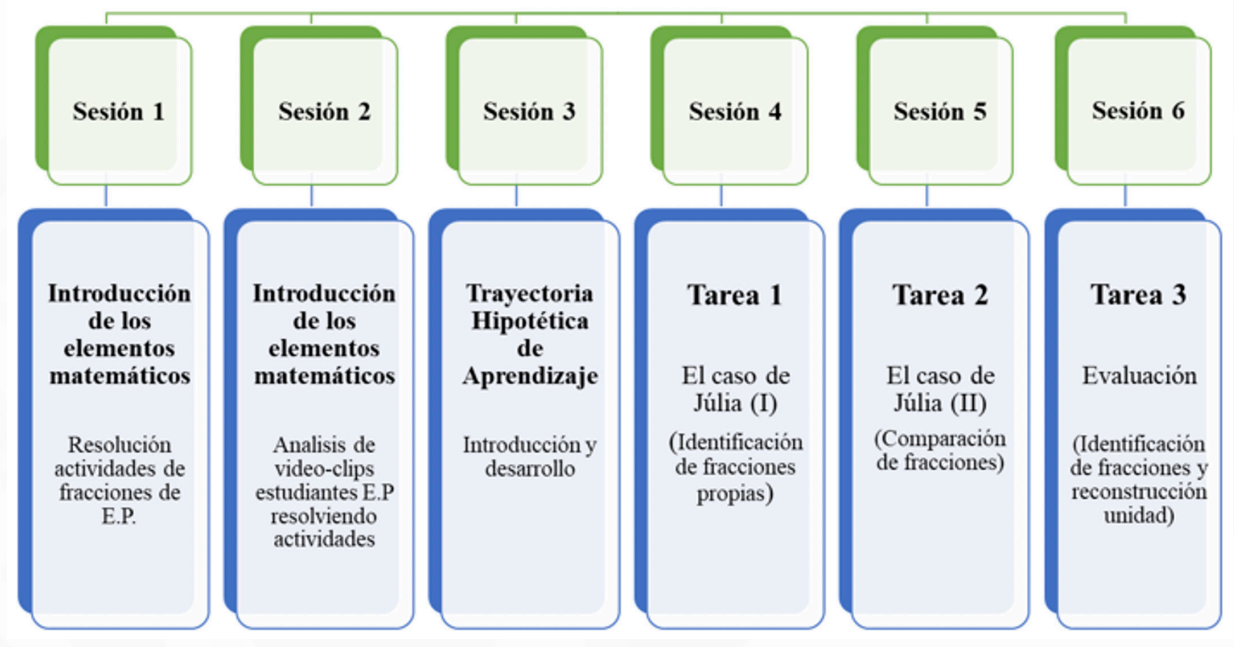

Figura 3. Distribución de las sesiones del entorno de aprendizaje centrado en el significado de fracción como parte-todo (Ivars, 2018, p. 85). 
La THA se proporciona como documento teórico e incluye: un camino hipotético de aprendizaje de los estudiantes de primaria (niveles de progresión, Tabla 1), ejemplos de respuestas de estudiantes de primaria que reflejan características de cada uno de los niveles de progresión (Figura 4) y ejemplos de actividades que podrían ayudar a los estudiantes a progresar en su comprensión del concepto de fracción como parte-todo (Tabla 2). Esta THA se diseña considerando resultados de investigaciones previas sobre la comprensión de la fracción como parte-todo de los estudiantes de educación primaria (Battista, 2012; Steffe y Olive, 2010). El objetivo de este documento teórico es proporcionar al futuro profesorado el conocimiento que necesita usar para identificar aspectos relevantes de los registros de la práctica, interpretarlos y apoyar sus decisiones de enseñanza. La información proporcionada en la THA integra conocimiento sobre las fracciones y conocimiento sobre la enseñanza y aprendizaje de las fracciones.

La Figura 4 muestra ejemplos de respuestas de estudiantes de primaria que reflejan características del nivel 2 de progresión. La respuesta del estudiante 1 muestra el uso de una parte (fracción unitaria, 1/4) como una unidad iterativa para representar la fracción propia 3/4. La respuesta del estudiante 2 refleja dificultades para representar fracciones impropias al no identificar la fracción unitaria 1/4 como unidad iterativa (una unidad para contar y poder representar 5/4). Esta es una característica de nivel 3.

\section{Tabla 1}

Niveles de progresión de la THA (Adaptado de Ivars, Fernández y Llinares, 2018a, p.271)

\begin{tabular}{|c|c|}
\hline Nivel 1 & $\begin{array}{l}\text { Los niños/as no pueden identificar y representar fracciones } \\
\text { - No reconocen que las partes en las que se divide el todo deben ser iguales en tamaño. } \\
\text { - No usan el mismo todo para comparar fracciones. }\end{array}$ \\
\hline Nivel 2 & $\begin{array}{l}\text { Los niños/as pueden identificar y representar fracciones propias } \\
\text { - Reconocen que las partes en las que se divide el todo deben ser iguales en tamaño, aunque no tengan la misma forma. } \\
\text { - Usan una fracción unitaria como una unidad iterativa para construir fracciones propias. } \\
\text { - No reconocen que una parte puede estar dividida en otras partes. } \\
\text { - Comparan fracciones usando el mismo todo. }\end{array}$ \\
\hline Nivel 3 & $\begin{array}{l}\text { Los niños/as pueden identificar y representar fracciones propias e impropias } \\
\text { - Reconocen que una parte puede estar dividida en otras partes. } \\
\text { - Usan cualquier fracción como unidad iterativa para construir fracciones propias e impropias. } \\
\text { - Reconocen que el tamaño de la parte disminuye cuando el número de partes aumenta. }\end{array}$ \\
\hline
\end{tabular}

La Tabla 2 muestra un ejemplo del tipo de actividad que aparece en el documento de la THA como actividad que podría ayudar a los estudiantes de primaria a progresar en su comprensión del significado de fracción como parte-todo (del nivel 2 al nivel 3). La actividad tiene como objetivo el uso de la fracción unitaria como unidad iterativa para construir la unidad y fracciones impropias usando las Regletas Cuisenaire. 


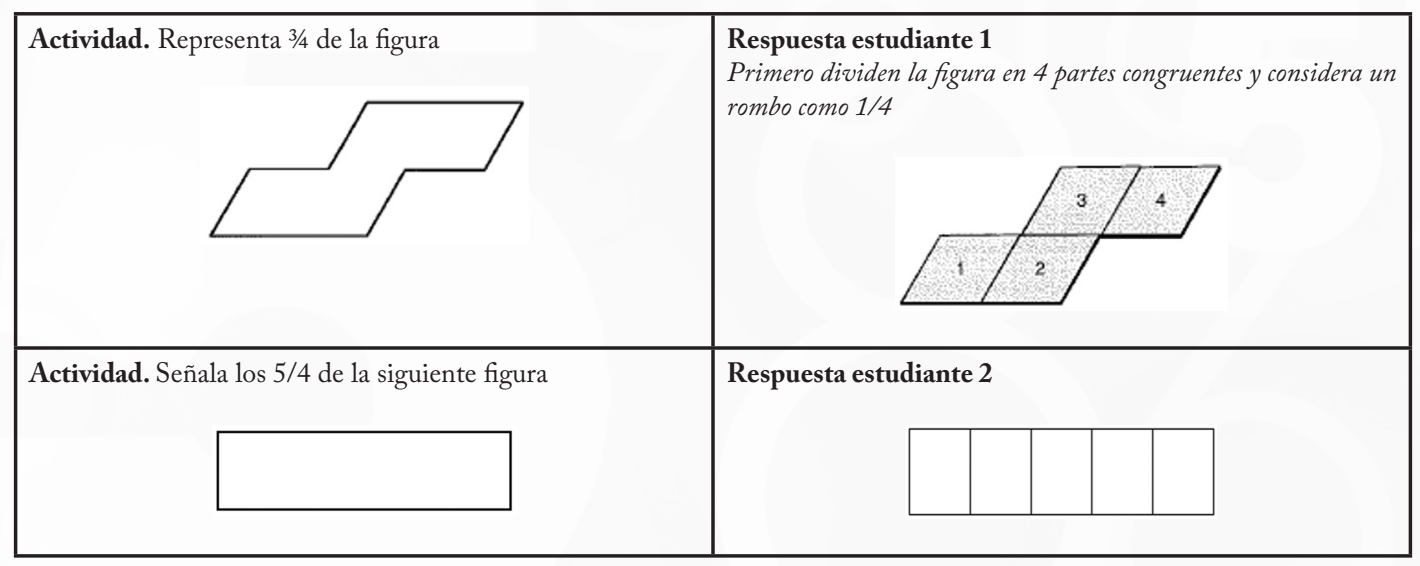

Figura 4. Registro de la práctica: Respuestas de estudiantes de primaria con características de nivel 2

\section{Tabla 2}

Ejemplo de actividad que podría ayudar a los alumnos a progresar en su comprensión del nivel 2 al 3

Objetivo: Usar una parte (la fracción unitaria) como una unidad iterativa para construir la unidad (el todo) y fracciones impropias.

Material: Regletas Cuisenaire.

Actividad 2A. Si la regleta roja es $1 / 3$ de la unidad ¿Qué regleta representa la unidad?

Actividad 2B. Si la regleta roja es $1 / 3$ de la unidad. ¿Qué fracción representa la regleta lila? ¿Y la marrón?

En el apartado siguiente se detallan las características de las viñetas usadas en los entornos de aprendizaje y se muestra un ejemplo de viñeta que corresponde a la tarea profesional 3 del entorno de aprendizaje descrito.

\section{Características de las Viñetas}

En las teorías de cognición situada (Brown, Collins y Duguid, 1989) se asume que el conocimiento es inseparable de los contextos y las actividades en los que se desarrolla. Esta perspectiva aboga por un aprendizaje en el que el futuro profesorado adquiera el conocimiento y competencias conectadas con situaciones de uso. Brown et al. (1989) argumentan que esta perspectiva sugiere el uso de actividades auténticas entendidas como: "las prácticas ordinarias de la cultura" (p. 34). Desde esta perspectiva, las viñetas entendidas como representaciones de la práctica se pueden considerar actividades auténticas. De acuerdo con Buchbinder y Kuntze (2018) una representación de la práctica puede representar un aspecto o varios aspectos de una situación de clase, pero no todas las características de una clase. Esta reducción de información hace que las viñetas sean instrumentos útiles en la formación de profesorado (Skilling y Styliniades, 2019) ya que permiten focalizar la atención de estos en aspectos sobre la práctica que son objeto de aprendizaje. 
Las viñetas proporcionan al profesorado contextos reales para analizar e interpretar aspectos de la enseñanza y aprendizaje de las matemáticas y por tanto proporcionan oportunidades para relacionar ideas teóricas sobre la enseñanza y aprendizaje de las matemáticas con ejemplos de la práctica (Buchbinder y Kuntze, 2018; Fernández et al., 2018). Se pueden diseñar diferentes formatos de viñetas (Friesen y Kuntze, 2018): grabaciones en vídeo de situaciones reales de aula (van Es y Sherin, 2008), respuestas de estudiantes a diferentes problemas o diálogos entre el maestro y los alumnos resolviendo diferentes problemas (Fernández et al., 2018; Ivars et al., 2020) o animaciones o cómic (Herbst y Kosko, 2014; Samkova, 2018)

Las viñetas de nuestros entornos de aprendizaje están formadas por respuestas de estudiantes de primaria/ secundaria a actividades con distintos grados de comprensión, por preguntas guía en relación con las tres destrezas que articulan la competencia docente: identificar los elementos matemáticos, interpretar la comprensión de los estudiantes y tomar decisiones para ayudar a los estudiantes a progresar en su comprensión, y por la información teórica que permite interpretar el registro de la práctica (respuestas de estudiantes con distintos grados de comprensión) y dar respuesta a las preguntas guía.

La viñeta que se presenta como ejemplo, está formada por dos actividades sobre fracciones (Figura 5), las respuestas de tres estudiantes de primaria que muestran diferentes características de la comprensión, es decir, estos estudiantes muestran características de los diferentes niveles de progresión mostrados en la THA (Figura 6) y por las siguientes tres cuestiones (preguntas guía) relacionadas con las destrezas de la competencia:

- Describe cómo ha resuelto cada estudiante la actividad, identificando cómo han utilizado los elementos matemáticos implicados y las dificultades que han tenido con ellos.

- ¿En qué nivel de la THA situarías a cada estudiante? Justifica tu respuesta.

- Teniendo en cuenta el nivel en el que has situado a cada estudiante, define un objetivo de aprendizaje y propón una actividad (o modifica la propuesta inicialmente por Júlia) para ayudar a los estudiantes a progresar en la comprensión de las fracciones según los niveles de progresión establecidos en la THA.

En la actividad 1 se tienen que identificar distintas representaciones de la fracción propia 3/8. Los estudiantes de primaria deben de tener en cuenta los elementos matemáticos: las partes en las que se divide el todo deben ser iguales en tamaño y una parte puede estar dividida en otras partes. En la actividad 2 se tiene que reconstruir la unidad a partir de una parte (en este caso la identificación de la fracción unitaria 1/3). Los estudiantes de primaria deben de tener en cuenta los elementos matemáticos: las partes en las que se divide el todo deben ser iguales en tamaño e identificar una de estas partes como una unidad iterativa (1/3) que permita construir la unidad (3/3). 


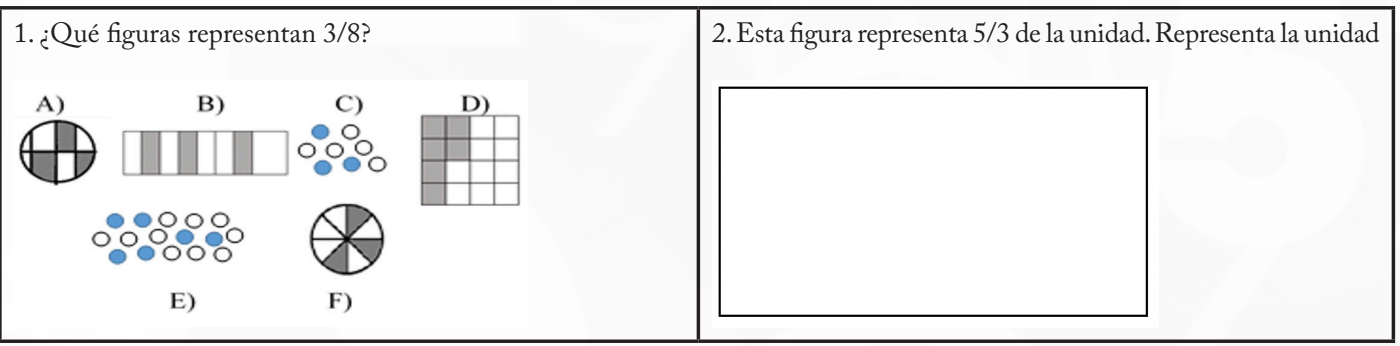

Figura 5. Actividades que forman parte de la viñeta (Adaptada de Ivars et al., 2018a, p. 273)

Con relación a las respuestas de los estudiantes (Figura 6), el estudiante 1 presenta dificultades para reconocer que las partes en las que se divide el todo deben ser iguales en tamaño en la actividad 1 y en la actividad 2, por lo que muestra características del nivel 1 de progresión de la THA. El estudiante 2 reconoce que las partes en las que se divide el todo deben ser iguales en tamaño tanto en la actividad 1 como en la 2 . Sin embargo, en la actividad 1 tienen dificultades para reconocer que una parte puede estar dividida en otras partes (al no reconocer D como una representación de 3/8) y en la actividad 2 tiene dificultades con la identificación de la fracción unitaria, es decir, no identifica que la figura proporcionada es 5 veces 1/3. Por tanto, el estudiante 2 muestra características del nivel 2 de progresión de la THA. Por último, el estudiante 3 es capaz de identificar las representaciones de la fracción 3/8 en la actividad 1 y es capaz de reconstruir el todo en la actividad 2. Este último estudiante muestra características del nivel 3 de progresión de la THA.

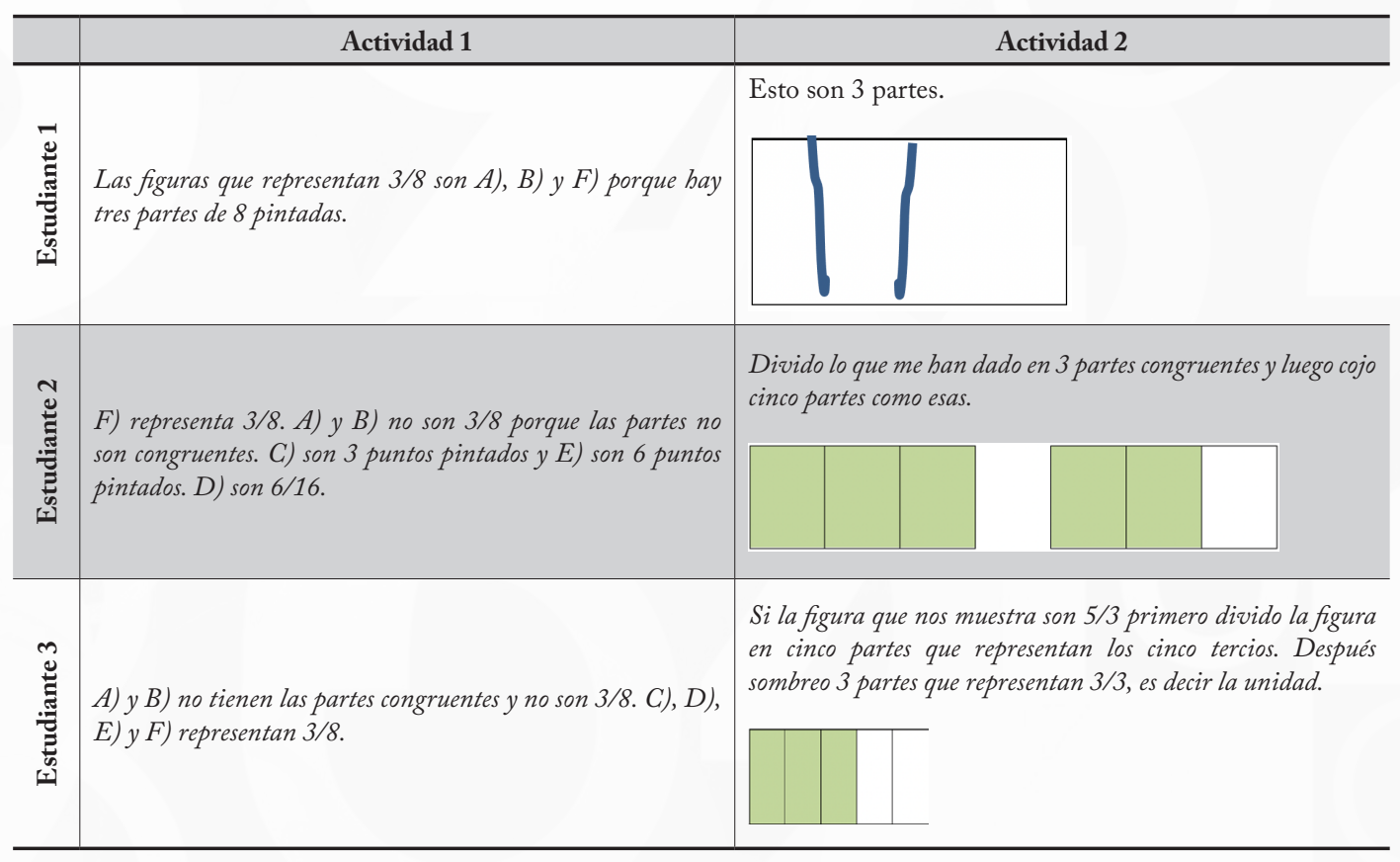

Figura 6. Respuestas de los tres estudiantes de primaria a las dos actividades que forman parte de la viñeta (Adaptada de Ivars et al., 2018a, p.273) 


\section{Resultados desde las Investigaciones}

Las investigaciones de GIDIMAT usando estos entornos de aprendizaje están permitiendo caracterizar grados de desarrollo de la competencia docente en el profesorado de educación infantil, primaria y secundaria. Es decir, grados de desarrollo sobre cómo el futuro profesorado de matemáticas está aprendiendo a usar el conocimiento en actividades profesionales.

De esta manera, en Sánchez-Matamoros, Moreno, Pérez-Tyteca y Callejo (2018) se muestra que, la instrumentación de una trayectoria hipotética de aprendizaje para un determinado concepto matemático (en la investigación el foco es la magnitud longitud y su medida en futuro profesorado de educación infantil) por parte del futuro profesorado es una evidencia del desarrollo de la competencia docente mirar profesionalmente el pensamiento matemático de los estudiantes. El uso progresivo de la THA a lo largo de un entorno de aprendizaje permite generar características del desarrollo de la competencia (Figura 7).

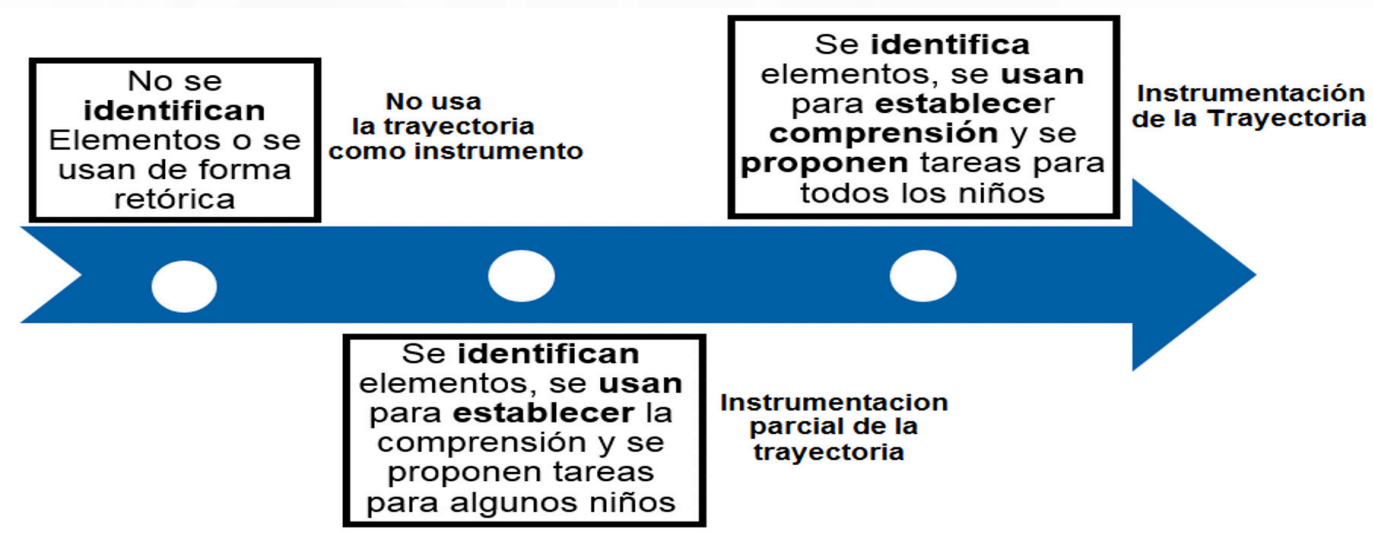

Figura 7. Características del desarrollo de la competencia mirar profesionalmente el pensamiento matemático (Traducida de Fernández et al.,2018, p. 50).

Por otra parte, el reconocimiento por parte del futuro profesorado de matemáticas del elemento matemático cuya comprensión determina un avance en el aprendizaje conceptual de los estudiantes (KDU, Key Developmental Understanding, Simon 2006) parece relevante en la caracterización del desarrollo de la competencia cuando se introduce la idea de THA como un referente para razonar sobre el pensamiento matemático de los estudiantes. Un KDU implica un avance conceptual por parte de los estudiantes, es decir, un cambio en la capacidad para pensar y/o percibir determinadas relaciones matemáticas (Simon, 2006, p. 362). En este sentido, si el futuro profesorado reconoce los KDU de un concepto matemático particular, será capaz de anticipar o interpretar cómo la comprensión de un determinado concepto se desarrolla (Buforn et al., 2020; Llinares, Fernández y Sánchez-Matamoros, 2016; Sánchez-Matamoros et al., 2015). 
Además, Ivars et al. (2018b) han mostrado que el progreso en el discurso profesional puede ser considerado una evidencia del desarrollo de la competencia docente mirar profesionalmente. En el entorno de aprendizaje descrito anteriormente, el futuro profesorado que elaboraba un discurso más detallado y específico sobre el pensamiento matemático de los estudiantes aportando evidencias para apoyar sus interpretaciones proporcionaba más decisiones de acción centradas en el progreso conceptual de los estudiantes. En este estudio se muestra que la THA ayudó al futuro profesorado de educación primaria a mejorar su discurso, entendiendo esta mejora como la elaboración de un discurso más detallado y específico cuando interpretaban la comprensión de los estudiantes (Figura 8). Elaborar un discurso más detallado sobre la comprensión de los estudiantes permitió al futuro profesorado diseñar actividades que ayudaran a los estudiantes a progresar en su aprendizaje. Por tanto, las THA son vistas como herramientas que apoyan el desarrollo de la competencia.

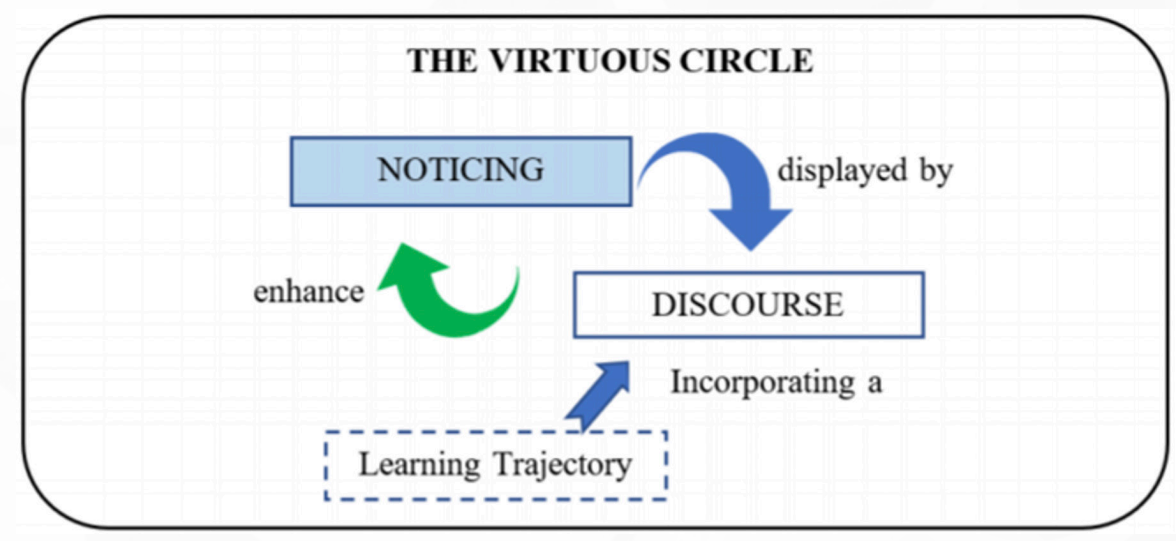

Figura 8. El "virtuous circle of noticing" en los programas de formación de profesorado (Ivars et al., 2018b, p. 13).

La generación de indicadores para caracterizar niveles de desarrollo de la competencia mirar profesionalmente la enseñanza de las matemáticas pone de manifiesto la relación entre la forma del discurso y su contenido. Por ejemplo, cómo se relacionan las evidencias desde el registro de la práctica con los elementos teóricos que permiten identificar lo relevante en un registro de la práctica e interpretarlo, y cómo se comprende el contenido matemático y la información en la THA que configura el contenido del discurso.

\section{Implicaciones y Trabajos Futuros}

Como formadores de profesores e investigadores, uno de nuestros objetivos es comprender cómo el futuro profesorado aprende a interpretar las situaciones de enseñanza-aprendizaje. Nuestras investigaciones han comenzado a generar descriptores del grado de desarrollo de la competencia mirar profesionalmente el pensamiento matemático de los estudiantes en los programas de formación inicial. Estos descriptores nos están permitiendo identificar trayectorias de aprendizaje del profesor. Las 
trayectorias de aprendizaje del profesor están caracterizadas de acuerdo a la articulación de las destrezas: atender, interpretar y decidir, y la relación entre estas. Sin embargo, más estudios son necesarios en esta línea analizando si es posible el diseño de módulos de formación basados en trayectorias de desarrollo de la competencia generadas empíricamente (Fernández y Choy, 2020; Fernández et al., 2018).

Por otra parte, el uso de otras herramientas distintas a las THA centradas en la planificación de lecciones podría complementar esta perspectiva. Por ejemplo, el constructo “curricular noticing” (Amador, Males, Earnest y Dietiker, 2017) que explora cómo el futuro profesorado mira profesionalmente las actividades/problemas, cómo secuencia lecciones y cómo selecciona los materiales curriculares que van a ser usados, o la noción de "affordances" usada por Choy y Didnyal (2017) para analizar lo que el futuro profesorado es capaz de atender de las características de las actividades/problemas y cómo aprovecha las posibilidades de estas actividades en el aula (Fernández y Choy, 2020).

Por otra parte, nuestras investigaciones están aportando información sobre las características que deben incluir los entornos de aprendizaje, y en particular, las viñetas para favorecer el desarrollo de la competencia mirar profesionalmente en los programas de formación. En este contexto, el objetivo principal del proyecto europeo coReflect@maths es desarrollar una herramienta digital (DIVER Tool - Designing and Investigating Vignettes for Education and Research Tool) que apoye al futuro profesorado a reflexionar colaborativamente usando representaciones de la práctica. Esta herramienta digital permitirá la creación fácil, tanto para el profesorado como para los formadores de profesores, de viñetas en formato de dibujos animados (cartoons) así como la integración de texto y vídeo (Krummenauer et al., 2020). Además, permitirá el desarrollo y la evaluación de cursos centrados en el uso de viñetas en formación inicial y de cursos dirigidos a formadores de profesores.

\section{Agradecimientos}

Esta investigación ha recibido el apoyo del proyecto I+D+i EDU2017-87411-R financiado por el Ministerio de Economía y Competitividad, España y el apoyo del Programa de la Unión Europea Erasmus+ (project coReflect@maths, 2019-1-DE01-KA203-004947). El apoyo de la Comisión Europea para la producción de esta publicación no constituye una aprobación del contenido, el cual refleja únicamente las opiniones de los autores, y la Comisión no se hace responsable del uso que pueda hacerse de la información contenida en la misma.

\section{Referencias}

Amador, J., Males, L. M., Earnest, D. y Dietiker, L. (2017). Curricular noticing: theory on practice of teachers' curricular use. En E. O. Schack, M. H. Fisher y J. A. Wilhelm (Eds.), Teacher Noticing: Bridging and broadening perspectives, contexts, and frameworks (pp. 427-444). Cham, Switzerland: Springer. 
Battista, M. T. (2012). Cognition-Based Assessment and teaching of fractions: Building on students' reasoning. Portsmouth, N.H. Heinemann.

Brown, J., Collins, A. y Duguid (1989). Situation cognition and the culture of learning. Educational Researcher, 18(1), 32-42.

Brown, L., Fernández, C., Helliwell, T. y Llinares, S. (2020). Prospective mathematics teachers as learners in university and school contexts. From university-based activities to classroom practice. En G. M. Lloyd y O. Chapman (Eds) International Handbook of Mathematics Teachers Education: Volume 3. Participants in Mathematics Teacher Education (vol. 13, pp. 343-366). Koninklijke Brill NV, Leiden.

Buchbinder, O. y Kuntze, S. (2018). Representations of practice in teacher education and research - Spotlights on Different Approaches. En O. Buchbinder y S. Kuntze (Eds.), Mathematics teachers engaging with representations of practice. A dynamically evolving field (pp. 1-8). Cham, Switzerland: Springer.

Buforn, A., Llinares, S., Fernández, C., Coles, A. y Brown, L. (2020). Pre-service teachers' knowledge of the unitizing process in recognizing students' reasoning to propose teaching decisions. International Journal of Mathematical Education in Science and Technology. doi.org/10.1080/0020739X.2020.1777333

Carrillo, J., Climent, N., Contreras, L. C. y Muñoz-Catalán, M. C. (2013). Determining specialised knowledge for mathematics teaching. En B. Ubuz, C. Haser y M. A. Mariotti (Eds.).Actas del CERME 8 (pp. 2985-2994). Middle East Technical University, Ankara, Turquía: ERME.

Choy, B. H. y Dindyal, J. (2017). Noticing affordances of a typical problem. En B. Kaur, W. K. Ho, T. L. Toh y B. H. Choy (Eds.), Proceedings of the 41st Conference of the International Group for the Psychology of Mathematics Education (vol. 2, pp. 249-256). Singapore: PME.

Clements D. H. y Sarama, J. (2009). Learning trajectories in early mathematics-sequences of acquisition and teaching. Encyclopedia of Language and Literacy Development, 1-7.

Design-Based Researcher Collective (2003). Design-Based Research: an emerging paradigm for educational inquiry. Educational Researcher, 32(1), 5-8.

Edgington, C., Wilson, P. H., Sztajn, P.y Webb, J. (2016). Translating learning trajectories into useable tools for teachers. Mathematics Teacher Educator, 5(1), 65-80. NV, Leiden.

Eraut, M. (1996). Developing professional knowledge and competence. London: The Falmer Press. 
Fernández, C. y Choy, B. H. (2020). Theoretical lenses to develop mathematics teacher noticing. Learning, Teaching, Psychological, and social perspectives. En S. Llinares y O. Chapman (Eds) International Handbook of Mathematics Teachers Education: Volume 2. Tools and Processes in Mathematics Teacher Education (vol. 12, pp. 337-360). Koninklijke Brill NV, Leiden.

Fernández, C., Llinares, S. y Rojas, Y. (2020). Prospective mathematics teachers' development of noticing in an online teacher education program. ZDM Mathematics Education, 52, 959-972.

Fernández, C., Llinares, S. y Valls, J. (2012). Learning to notice students' mathematical thinking through on-line discussions. ZDM. Mathematics Education, 44, 747-759.

Fernández, C., Sánchez-Matamoros, G., Moreno, M. y Callejo, M. L. (2018). La coordinación de las aproximaciones en la comprensión del concepto de límite cuando los estudiantes para profesor anticipan respuestas de estudiantes. Enseñanza de las Ciencias, 36(1), 143-162.

Fernández, C., Sánchez-Matamoros, G., Valls, J. y Callejo, M. L. (2018). Noticing students' mathematical thinking: characterization, development and contexts. AIEM. Avances en Investigación Matemática, 13, 39-61.

Friesen, M. y Kuntze, S. (2018). Competence assessment with representations of practice in text, comic and video format. En O. Buchbinder y S. Kuntze (Eds.), Mathematics teachers engaging with representations of practice. A dynamically evolving field (pp. 113-130). Cham, Switzerland: Springer.

Godino, J. D. Batanero, C. y Font, V. (2007). The onto-semiotic approach to research in mathematics education. ZDM. The International Journal on Mathematics Education, 39 (1-2), 127-135.

Goodwin, C. (1994). Professional vision. American Anthropologist, 96, 606-633.

Herbst, P.y Kosko, K.W. (2014). Using representations of practice to elicit mathematics teachers' tacit knowledge of practice: a comparison of responses to animations and videos. Journal of Mathematics Teacher Education, 17(6), 515-537.

Hill, H. C., Ball, D. L. y Schilling, S. G. (2008). Unpacking pedagogical content knowledge: Conceptualizing and measuring teachers' topic-specific knowledge of students. Journal for Research in Mathematics Education, 39(4), 372-400.

Ivars, P. (2018). Mirar profesionalmente el pensamiento matemático de los estudiantes: El esquema fraccionario (Tesis Doctoral). Universidad de Alicante. 
Ivars, P., Buforn À. y Llinares, S. (2017). Diseño de tareas y desarrollo de una mirada profesional sobre las situaciones de enseñanza de las matemáticas de estudiantes para maestro. En A. Salcedo, (Comp.). Alternativas Pedagógicas para la Educación Matemática del siglo XXI (pp. 65 - 87). Caracas: Centro de Investigaciones Educativas, Escuela de Educación. Universidad Central de Venezuela.

Ivars, P. y Fernández, C. (2018). The role of writing narratives in developing pre-service elementary teachers' noticing. En G. Stylianides y K. Hino (Eds.), Research Advances in the Mathematical Education of Pre-service Elementary Teachers. ICME-13 Monographs. Hamburg, Germany: Springer.

Ivars, P., Fernández, C. y Llinares, S. (2018a). Características del desarrollo de la competencia mirar profesionalmente el pensamiento de los estudiantes sobre fracciones. En L. J. Rodríguez-Muñiz, L. Muñiz-Rodríguez, A. Aguilar-González, P. Alonso, F. J. García García y A. Bruno (Eds.), Investigación en Educación Matemática XXII (pp. 270-279). Gijón: SEIEM.

Ivars, P., Fernández, C. y Llinares, S. (2020). A Learning Trajectory as a scaffold for pre-service teachers' noticing of students' mathematical understanding. International Journal of Science and Mathematics Education, 18, 529-548.

Ivars, P., Fernández, C., Llinares, S. y Choy, B. H. (2018b). Enhancing noticing: Using a hypothetical learning trajectory to improve pre-service primary teachers' professional discourse. Eurasia. Journal of Mathematics, Science and Technology Education, 14(11), em1599.

Jacobs, V. R., Lamb, L. L. y Philipp, R. A. (2010). Professional noticing of children's mathematical thinking. Journal for Research in Mathematics Education, 41(2), 169-202.

Krummenauer et al. (2020). Developing a digital tool for vignette-based professional development of mathematics teachers - The potential of different vignette formats. Tenth ERME Topic Conference (ETC10) on Mathematics Education in the Digital Age (MEDA).

Llinares, S. (2004). La generación y uso de instrumentos para la práctica de enseñar matemáticas en Educación Primaria. UNO, Revista de Didáctica de la Matemática, 36, 93-115.

Llinares, S. (2013). Professional noticing: A component of the mathematics teacher's professional practice. Sisyphus-Journal of Education, 1(3), 76-93.

Llinares, S. (2014). Experimentos de enseñanza e investigación. Una dualidad en la práctica del formador de profesores de matemáticas. Educación Matemática, no extraordinario, marzo, 31-51. 
Llinares, S. (2019). Indicators for the development of noticing: how do we recognize them? For the Learning of Mathematics, November, 38-43.

Llinares, S., Fernández, C. y Sánchez-Matamoros, G. (2016). Changes in how prospective teachers anticipate secondary students' answers. Eurasia Journal of Mathematics, Science Eซ Technology Education, 12(8), 2155-2170.

Llinares, S., Ivars, P., Buforn, A. y Groenwald, C. (2019). “Mirar profesionalmente” las situaciones de enseñanza una competencia basada en el conocimiento. En E. Badillo, N. Climent, C. Fernández y M. T. González (Eds.), Investigación sobre el profesor de matemáticas: formación, práctica de aula, conocimiento y competencia profesional (pp. 177-192). Salamanca: Ediciones Universidad Salamanca.

Llinares, S. y Valls, J. (2010). Prospective primary mathematics teachers'learning from on-line discussions in a virtual video-based environment. Journal of Mathematics Teacher Education, 13, 177-196.

Lobato, J.y Walters, C. D. (2017). A taxonomy of approaches to learning trajectories and progressions. En J. Cai (Ed.), Compendium for research in mathematics education (pp.74-101). Reston, VA: National Council of Teachers of Mathematics.

Mason, J. (2002). Researching your own practice. The discipline of noticing. London: Routledge-Falmer.

National Council of Teachers of Mathematics. (2014). Principles to actions: Ensuring mathematics success for all. Reston, VA: NCTM

Roller, S.A. (2016). What they notice in video: A study of prospective secondary mathematics teachers learning to teach. Journal of Mathematics Teacher Education, 19(5), 477-498.

Rowland, T., Huckstep, P. y Thwaites, A. (2005). Elementary teachers' mathematics subject knowledge: The knowledge quartet and the case of Naomi. Journal of Mathematics Teacher Education, $8(3), 255-281$.

Samkova, L. (2018). Concept cartoons as a representation of practice. En O. Buchbinder y S. Kuntze (Eds.), Mathematics teachers engaging with representations of practice. A dynamically evolving field (pp. 71-93). Cham, Switzerland: Springer

Sánchez-Matamoros, G., Fernández, C.y Llinares, S. (2015). Developing pre-service teachers' noticing of students' understanding of the derivative concept. International Journal of Science and Mathematics Education, 13(6), 1305-1329. 
Sánchez-Matamoros, G., Fernández, C. y Llinares, S. (2019). Relationships among prospective secondary mathematics teachers' skills of attending, interpreting and responding to students' understanding. Educational Studies in Mathematics, 100(1), 83-99

Sánchez-Matamoros, G., Moreno, M., Pérez-Tyteca, P. y Callejo, M. L. (2018). Trayectoria de aprendizaje de la longitud y su medida como instrumento conceptual usado por futuros maestros de educación infantil. Revista Latinoamericana de Investigación en Matemática Educativa, 21(2), 203-228

Schack, E. O., Fisher, M. H. y Wilhelm, J. A. (Eds.). (2017). Teacher noticing: Bridging and broadening perspectives, contexts, and frameworks. Cham, Switzerland: Springer.

Shulman, L. S. (1986). Those who understand: Knowledge growth in teaching. Educational Review, $57(1), 1-22$.

Simon, M. A. (1995). Reconstructing mathematics pedagogy from a constructivist perspective. Journal for Research in Mathematics Education, 114-145.

Simon, M. (2006). Key Developmental Understanding in mathematics: A direction for investigating and establishing learning goals. Mathematical Thinking and Learning, 8(4), 359-371.

Skilling, K. y Stylianides, G. (2019). Using vignettes in educational research: a framework for vignette construction. International Journal of Research and Method in Education.

Stahnke, R., Schueler, S. y Roesken-Winter, B. (2016). Teachers' perception, interpretation, and decisionmaking: a systematic review of empirical mathematics education research. $Z D M, 48(1-2), 1-27$.

Steffe, L. y Olive, J. (2009). Children's fractional knowledge. Springer Science \& Business Media.

Sztajn, P., Confrey, J., Wilson, P.H. y Edgington, C. (2012). Learning trajectory based instruction: Toward a theory of teaching. Educational Researcher, 41(5), 147-156.

Sztajn, P. y Wilson, P. H. (2019). Learning trajectories for teachers: Designing effective professional development for math instruction. Nueva York, NY: Teachers' College Press.

Thomas, J., Jong, C., Fisher, M. H. y Schack, E. O. (2017). Noticing and knowledge. Exploring Theoretical connections between professional noticing and mathematical knowledge for teaching. The Mathematics Educator, 26(2), 3-25. 
van Es, E. A. y Sherin, M. G. (2002). Learning to notice: Scaffolding new teachers' interpretations of classroom interactions. Journal of Technology and Teacher Education, 10(4), 571-595.

van Es, E. A. y Sherin, M. G. (2008). Mathematics teachers' "learning to notice" in the context of a video club. Teaching and Teacher Education, 24(2), 244-276.

Wilson, P. H., Sztajn, P., Edgington, C. y Myers, M. (2015). Teachers' uses of a learning trajectory in student-centered instructional practices. Journal of Teacher Education, 66(3), 227-244. 